\title{
A NEW DEFINITION OF A REDUCED FORM
}

\author{
E. H. HADLOCK AND T. O. MOORE
}

Introduction. Let a ternary quadratic form $f=a x^{2}+b y^{2}+c z^{2}+2 r y z$ $+2 s x z+2 t x y$, with integral coefficients, be denoted by $f=[a, b, c, r$, $s, t]$. Associated with $f$ is the determinant $d(\neq 0)$ defined by

$$
d=\left|\begin{array}{lll}
a & t & s \\
t & b & r \\
s & r & c
\end{array}\right|
$$

We consider in this paper indefinite forms (forms which represent both positive and negative integers) as well as positive forms (those which represent only nonnegative integers). All number symbols which appear in this paper represent integers.

In [3] it is shown that every primitive ternary quadratic form is equivalent to a primitive form in which the coefficients of $2 x z$ and $2 x y$ are one and zero respectively. In this present paper we find that any ternary quadratic form $f_{z}$ is equivalent to a form $f=[a, b, c, r, s, t]$ in which $a$ (or $-a$ ) is the minimum nonzero integer represented by $\left|f_{2}\right|$ (called the first minimum; $b$ (or $-b$ ) is the second minimum represented by $f_{2} ; c$ (or $-c$ ) is the third minimum represented by $f_{2}$ ). (See definitions for second and third minimums.) Finally either $t \mid(|a|, d)$ and $s=0$ or $t=0$ and $s \mid(|a|, d)$. Thus our definition of a reduced form is quite simple. (See definition of a reduced form and conventions adopted to insure uniqueness.)

Theorem 1. Let $f_{2}=\left[a_{2}, b_{2}, c_{2}, r_{2}, s_{2}, t_{2}\right]$ be a form. Let

$$
m_{1}=\min \left\{\left|f_{2}(x, y, z)\right|: f_{2}(x, y, z) \neq 0\right\} \text {. }
$$

Then $f_{2}$ is equivalent to a form $f_{1}=\left[a, b_{1}, c_{1}, r_{1}, s_{1}, t_{1}\right]_{\ni} m_{1}=|a|, a$ is represented primitively by $f_{2}$ and $g_{1} \mid g$ where

$$
g_{1}=\left(a, t_{1}, s_{1}\right) \text { and } g=(a, d) .
$$

Proof [1]. Let $a=f_{2}\left(k_{1}, l_{1}, n_{1}\right)$ where $m_{1}=\left|f_{2}\left(k_{1}, l_{1}, n_{1}\right)\right|$. Then $\left(k_{1}, l_{1}, n_{1}\right)=1$, for, if $\left(k_{1}, l_{1}, n_{1}\right)=g_{2}>1$, then $f_{2}\left(k_{1} / g_{2}, l_{1} / g_{2}, n_{1} / g_{2}\right)$ $=a / g_{2}$. But $|a| / g_{2}<|a|$, contradicting the definition of $a$. Hence $a$ is represented primitively by $f_{2}$. Further if $g_{3}=\left(k_{1}, l_{1}\right)$, then $\left(g_{3}, n_{1}\right)=1$ and there exist integers $k_{2}, l_{2}, k_{3}$ and $n_{3}, k_{1} l_{2}-l_{1} k_{2}=g_{3}$, and $g_{3} n_{3}-n_{1} k_{3}$ $=1$. Then the transformation

Received by the editors September 17, 1968. 


$$
T^{\prime}=\left(\begin{array}{ccc}
k_{1} & k_{2} & k_{1} k_{3} / g_{3} \\
l_{1} & l_{2} & l_{1} k_{3} / g_{3} \\
n_{1} & 0 & n_{3}
\end{array}\right)
$$

has determinant $\left|T^{\prime}\right|=1$, since

$$
\begin{aligned}
& n_{3}\left(k_{1} l_{2}-k_{2} l_{1}\right)+n_{1} k_{3} / g_{3}\left(k_{2} l_{1}-k_{1} l_{2}\right) \\
& =\left(g_{3} n_{3}-n_{1} k_{3}\right)\left(k_{1} l_{2}-k_{2} l_{1}\right) / g_{3}=1 .
\end{aligned}
$$

Thus $T^{\prime}$ applied to $f_{2}$ gives an equivalent form. Also, this equivalent form is $f_{1}$ having $a$ as the coefficient of $x^{2}$.

To show that $g_{1} \mid g$, observe that $a A+t_{1} T_{1}+s_{1} S_{1}=d$, where $A, T_{1}$ and $S_{1}$ are the cofactors of $a, t_{1}$ and $s_{1}$ respectively. Thus by (2) $g_{1} \mid d$ so that $g_{1} \mid g$.

Definitions. Let $\Sigma$ be defined by

$$
\Sigma=\left\{(x, y, z): a x+t_{1} y+s_{1} z=0, g \text { or }-g\right\},
$$

where $g$ is defined in (2) and $a, t_{1}$, and $s_{1}$ in Theorem 1. Define $a^{\prime}, t^{\prime}$ and $s^{\prime}$ by

(5) $a^{\prime}=a / g_{1}, \quad t^{\prime}=t_{1} / g_{1}, \quad s^{\prime}=s_{1} / g_{1}, \quad$ where $\quad\left(a^{\prime}, t^{\prime}, s^{\prime}\right)=1$,

and $g_{1}$ is defined in (2).

Theorem 2. Let $f_{1}=\left[a, b_{1}, c_{1}, r_{1}, s_{1}, t_{1}\right], m, g_{1}$ be given as in Theorem 1 and $a^{\prime}, t^{\prime}, s^{\prime}$ by (5). Then $f_{1}$ is equivalent to $f=[a, b, c, r, s, t]$ where

$$
|t|=g_{1} \text { and } s=0 .
$$

PROOF. If $s_{1} t_{1}=0$, then replacing $x$ by $x+h_{1} y_{1}+h_{2} z_{1}$ in $f_{1}$ for some $h_{1}$ and $h_{2}$ gives an equivalent form with the same leading coefficient $a$ and with the coefficients of $2 x z_{1}$ and $2 x y_{1}$ not zero. Thus we may assume that $s_{1} t_{1} \neq 0$ in $f_{1}$.

We apply the transformation

$$
T=\left(\begin{array}{lll}
1 & \rho & \sigma \\
0 & \alpha & \beta \\
0 & \gamma & \delta
\end{array}\right),|T|=1
$$

to $f_{1}$. Then

$$
\begin{aligned}
& a=f_{1}(1,0,0), \quad b=f_{1}(\rho, \alpha, \gamma), \quad c=f_{1}(\sigma, \beta, \delta), \\
& t=a_{\rho}+t_{1} \alpha+s_{1} \gamma, \quad s=a \sigma+t_{1} \beta+s_{1} \delta .
\end{aligned}
$$

We show first that there exists a triple $(x, y, z)=(\rho, \alpha, \gamma)$ satisf ying (4) with $t= \pm g_{1}$ and with $(\alpha, \gamma)=1$. Let 
(9)

$$
k^{\prime}=t^{\prime} \alpha+s^{\prime} \gamma
$$

Then by (5), (8), with $t= \pm g_{1}$

$$
k^{\prime}= \pm 1-a^{\prime} \rho .
$$

If $d_{1}=\left(t^{\prime}, s^{\prime}\right)$, then

$$
t^{\prime}=d_{1} t^{\prime \prime}, \quad s^{\prime}=d_{1} s^{\prime \prime}, \text { where }\left(t^{\prime \prime}, s^{\prime \prime}\right)=1 .
$$

Thus we must show that there exists a triple $(\rho, \alpha, \gamma)$ э

$$
k^{\prime}=d_{1}\left(t^{\prime \prime} \alpha+s^{\prime \prime} \gamma\right)= \pm 1-a^{\prime} \rho, \text { with }(\alpha, \gamma)=1 .
$$

Since $\left(a^{\prime}, t^{\prime}, s^{\prime}\right)=1$ then

$$
\left(d_{1}, a^{\prime}\right)=1 .
$$

Hence by (13) there exists an integer $\rho_{7}$

$$
a^{\prime} \rho \equiv \pm 1\left(\bmod d_{1}\right), \text { i.e., } \pm 1-a^{\prime} \rho=d_{1} \theta
$$

for some integer $\theta$. All solutions of (14) are given by

$$
\rho=\rho_{0}+d_{1} \theta^{\prime} \rightarrow \pm 1-a^{\prime} \rho_{0}=d_{1} \theta_{0},
$$

where $\rho_{0}, \theta_{0}$ is any solution of $(14)_{2}$ and $\theta^{\prime}$ is an arbitrary integer. By

$$
\pm 1-a^{\prime}\left(\rho-d_{1} \theta^{\prime}\right)=d_{1} \theta_{0} \Rightarrow \pm 1-a^{\prime} \rho=d_{1}\left(\theta_{0}-a^{\prime} \theta^{\prime}\right) .
$$

By $(15)_{2}\left(a^{\prime}, \theta_{0}\right)=1$. Thus by Dirichlet's theorem there exists $a \theta^{\prime}{ }_{3}$

$$
\theta_{0}-a^{\prime} \theta^{\prime}=d_{2}
$$

where $d_{2}$ is a prime and $\Rightarrow$

$$
\left(d_{2}, a^{\prime}\right)=1 .
$$

By $(11)_{2}$ there exists integers $\alpha$ and $\gamma_{7}$

$$
t^{\prime \prime} \alpha+s^{\prime \prime} \gamma=d_{2}
$$

and

$$
(\alpha, \gamma)=1 \text {. }
$$

(Suppose a prime $p \mid(\alpha, \gamma)$. Then $p=d_{2}$. By taking $\phi_{9}\left(\phi, d_{2}\right)=1$, we obtain $\alpha^{\prime}=\alpha+s^{\prime \prime} \phi, \gamma^{\prime}=\gamma-t^{\prime \prime} \phi$ as a solution of (19) with $\left(\alpha^{\prime}, \gamma^{\prime}\right)=1$. For if $\left(\alpha^{\prime}, \gamma^{\prime}\right)>1$, then $\left(\alpha^{\prime}, \gamma^{\prime}\right)=d_{2}$. Thus $d_{2} \mid s^{\prime \prime} \phi$ and $d_{2} \mid t^{\prime \prime} \phi$ which contradicts $(11)_{2}$ since $\left(\phi, d_{2}\right)=1$.) Hence by $(16)_{2},(17)$ and (19) there exists a triple $(\rho, \alpha, \gamma)$ satisfying $(12)_{2}$ with $(\alpha, \gamma)=1$. Thus by (12) we note that

$$
\left(k^{\prime}, a^{\prime}\right)=1 \text {. }
$$


In view of $(7)_{2}$ we want to find $\delta$ and $\beta$,

$$
\alpha \delta-\beta \gamma=1 \text {. }
$$

All solutions of (22) are given by

$$
\delta=\delta_{0}-\gamma n, \quad \beta=\beta_{0}-\alpha n,
$$

where $\delta_{0}, \beta_{0}$ is any solution of (22) and $n$ is an arbitrary integer. Thus by $(5),(6)_{2},(8)_{5},(9)$ and $(23)$

$$
a^{\prime} \sigma-k^{\prime} n=s / g_{1}-\left(t^{\prime} \beta_{0}+s^{\prime} \delta_{0}\right), \quad s=0 .
$$

Hence it is seen by (21), that (24) has a solution in $\sigma$ and $n$. Any such solution gives a triple $(\sigma, \beta, \delta) \rightarrow(24)$ holds, and (8) $)_{5}$ with $s=0$ also holds. [In fact all solutions of (24) are given by

$$
\sigma=\sigma_{0}-k^{\prime} k, \quad n=n_{0}-a^{\prime} k
$$

where $\sigma_{0}, n_{0}$ is any solution of (24) and $k$ is an arbitrary integer. Thus by (23) and (25) we determine $\beta$ and $\delta$ by

$$
\left.\beta=\beta_{0}-\alpha n_{0}+\alpha a^{\prime} k, \quad \delta=\delta_{0}-\gamma n_{0}+\gamma a^{\prime} k .\right]
$$

REMARKS. The transformation which replaces $y$ by $-z$ and $z$ by $y$ applied to $f$ of Theorem 2 gives a new form in which $a$ remains unchanged, the coefficient of $2 x z$ is $\pm g_{1}$ and the coefficient of $2 x y$ is zero. However we give Theorems 3 and 4 for the purpose of numerical calculation.

Theorem 3. Let $f_{1}=\left[a, b_{1}, c_{1}, r_{1}, s_{1}, t_{1}\right], m_{1}, g_{1}$ be given as in Theorem $1 ; a^{\prime}, t^{\prime}, s^{\prime}$ by (5). Let $\sigma$ and $n$ satisfy

$$
a[\alpha(\sigma+\rho n)-\rho \beta]=\alpha s-s_{1}, \quad \text { for some } \beta,
$$

where $(\rho, \alpha, \gamma)$ is some triple satisfying $(8)_{4}$ with $t=0,(\alpha, \gamma)=1$ and $\alpha \neq 0$, then there exists a transformation $T$ in (7) which transforms $f_{1}$ into the equivalent form $f=[a, b, c, r, s, t]$ where

$$
\boldsymbol{t}=\mathbf{0} \text { and }|s|=g_{1} \text {. }
$$

Proof [2]. Let $(x, y, z)=(\rho, \alpha, \gamma)$ in (3) 1 . Then all solutions of $(8)_{4}$ with $t=0$ are given by

$$
\rho=t^{\prime} w-s^{\prime} v, \quad \alpha=s^{\prime} u-a^{\prime} w, \quad \gamma=a^{\prime} v-t^{\prime} u,
$$

where $u, v$, and $w$ are arbitrary integers. Let

$$
d_{3}=\left(a^{\prime}, s^{\prime}\right), d_{4}=\left(a^{\prime}, t^{\prime}\right) ; \text { then }\left(a^{\prime} / d_{3}, s^{\prime} / d_{3}\right)=\left(a^{\prime} / d_{4}, t^{\prime} / d_{4}\right)=1 .
$$

Then by (29) 2 and (29) 


$$
\alpha / d_{3}=s^{\prime} / d_{3} u-a^{\prime} / d_{3} w, \quad \gamma / d_{4}=a^{\prime} / d_{4} v-t^{\prime} / d_{4} u .
$$

Let $u(\neq 0)$ be any fixed integer $\ni\left(u, a^{\prime}\right)=1$. Then by Dirichlet's theorem there exist integers $w$ and $v \ni$

$$
\alpha=d_{3} p_{1}, \quad \gamma=d_{4} p_{2},
$$

where $p_{1}$ and $p_{2}$ are primes and

$$
\left(p_{1}, d_{4}\right)=\left(p_{2}, p_{1}, d_{3}\right)=1 .
$$

But

$$
\left(d_{3}, d_{4}\right)=1 .
$$

For if a prime $p \mid d_{3}$ and $p \mid d_{4}$, then $p \mid\left(a^{\prime}, t^{\prime}, s^{\prime}\right)$ contradicting (5) $)_{4}$. Thus by (29), (31)-(33), there exist a triple $(\rho, \alpha, \gamma)$ satisfying $(8)_{4}$ with $t=0$ and $(\alpha, \gamma)=1$. We now seek a solution of (24) with $|s|=g_{1}$. Since $(\alpha, \gamma)=1$ and $\alpha \neq 0$, let $\beta, \delta$ satisfy (22). Hence

$$
\alpha \delta=1+\beta \gamma .
$$

Since $t=0$, then (10) is replaced by

$$
k^{\prime}=-a^{\prime} \rho \text {. }
$$

Thus by (5), (9). (24), (25), (34) and (35), $\sigma$ and $n$ must satisfy

$$
\begin{aligned}
a^{\prime} \alpha(\sigma+\rho n) & =\alpha s / g_{1}-\left(\alpha t^{\prime} \beta+\alpha \delta s^{\prime}\right)=\alpha s / g_{1}-\left(\alpha t^{\prime} \beta+s^{\prime}+\beta s^{\prime} \gamma\right) \\
& =\alpha s / g_{1}-s^{\prime}-\beta\left(t^{\prime} \alpha+s^{\prime} \gamma\right)=\alpha s / g_{1}-s^{\prime}+a^{\prime} \beta \rho .
\end{aligned}
$$

Since (27) holds, the desired transformation in (7) exists.

TheOREM 4. Let $f_{1}=\left[a, b_{1}, c_{1}, r_{1}, s_{1}, t_{1}\right], m_{1} g_{1}$ be given as in Theorem 1 and $a^{\prime}, t^{\prime}, s^{\prime}$ by (5). Let $d_{3}$ and $d_{4}$ be given by (30). Let $\rho$ and $\beta$ satisfy (36) $a^{\prime} \rho=t / g_{1}-s^{\prime} \gamma \quad$ and $t^{\prime} \beta \equiv s / g_{1}\left(\bmod d_{3}\right) \quad$ with $\beta \gamma=-1$ or $\rho$ and $\delta$ satisfy

$$
a^{\prime} \rho=t / g_{1}-t^{\prime} \alpha \quad \text { and } \quad s^{\prime} \delta \equiv s / g_{1}\left(\bmod d_{4}\right) \quad \text { with } \alpha \delta=1,
$$

where $|t|=g_{1}$ and $s=0$ or $t=0$ and $|s|=g_{1}$, then there exists a transformation (7) which transforms $f_{1}$ into the equivalent form $f=[a, b, c, r$, $s, t] \rightarrow$

$$
|t|=g_{1} \text { and } s=0 \text { or } t=0 \text { and }|s|=g_{1} \text {. }
$$

Proof. Suppose (36) holds. Let $\alpha=0$ and $(\rho, \beta, \gamma)$ satisfy (36). Since $\beta \gamma=-1,(7)_{2}$ holds. By (36) we observe that $(8)_{4}$ is satisfied. By $(36)_{2}$ let $\sigma$ and $\delta$ satisfy 


$$
a^{\prime} \sigma+s^{\prime} \delta=s / g_{1}-t^{\prime} \beta
$$

Thus $(8)_{4}$ holds.

Suppose (37) holds. Let $\gamma=0$ and $(\rho, \alpha, \delta)$ satisfy (37). Since $\alpha \delta=1$, $(7)_{2}$ holds. By $(37)_{1}$, we observe that $(8)_{4}$ is satisfied. By $(37)_{2}$ let $\sigma$ and $\beta$ satisfy $a^{\prime} \sigma+t^{\prime} \beta=s / g_{1}-s^{\prime} \delta$. Thus (8) $)_{5}$ holds.

Definitions. Let $J=\{T:|T|=1$ and $T$ transforms a given form $f_{2}$ of Theorem 1 into $f=[a, b, c, r, s, t] \Rightarrow a= \pm m_{1}$ of Theorem 1 and either $t \mid g$ and $s=0$ or $t=0$ and $s \mid g\}$.

(i) $m_{1}=\min \left\{\left|f_{2}(x, y, z)\right|: f_{2}(x, y, z) \neq 0\right\}$ is called the first minimum of $f_{2}$.

(ii) $m_{2}=\min \{|b|: T \in J\}$ is called the second minimum of $f_{2}$.

(iii) Let $J_{1}=\left\{T: T \in J\right.$ and $\left.|b|=m_{2}\right\}$. Then $m_{3}=\min \left\{|c|: T \in J_{1}\right\}$ is called the third minimum of $f_{2}$.

THEOREM 5. Let the form $f_{1}$ of Theorem 1 be given in which the coeffcient a of $x^{2}$ satisfies $a= \pm m_{1}$. Let $g_{1}=\left(a, t_{1}, s_{1}\right)$. Then $f_{1}$ is equivalent to $f=[a, b, c, r, s, t]$ where $b= \pm m_{2}, c= \pm m_{3}$, and where either $|t|=g_{1}$ and $s=0$ or $t=0$ and $|s|=g_{1}$.

Proof. By Theorem 2 there exist transformations which transform $f_{1}$ into forms $f=[a, b, c, r, s, t]_{\epsilon} a= \pm m_{1}$ and either $|t|=g_{1}$ and $s=0$ or $t=0$ and $|s|=g_{1}$. Let $J_{1}$ be those transformations for which $b= \pm m_{2}$. Then let $T \in J_{1}, c= \pm m_{3}$. Thus $T$ transforms $f_{1}$ into the desired form $f$.

Definition. A form $f=[a, b, c, r, s, t]$ is said to be reduced if $a= \pm m_{1}, b= \pm m_{2}, c= \pm m_{3}, r>0$ and either

$$
t \mid g \text { and } s=0 \text { or } s \mid g \text { and } t=0,
$$

where the following conventions are adopted:

(i) If $f=m_{1}$ and $f=-m_{1}$, then we choose $a=m_{1}$. Having fixed the sign of $a$, we do likewise for the sign of $b$ if such a choice exists. Next the sign of $c$ is determined as positive if a choice exists.

(ii) We choose the smallest possible divisors $|t|$ and $|s|$ of $g$, subject to (i).

(iii) If $f_{2}$ is equivalent to $f$ where $t \mid g$ and $s=0$ and also to $f$ where $s \mid g$ and $t=0$, we choose the latter as the reduced form provided (i) and (ii) are satisfied.

REMARKs. If $f=[a, b, c, r, s, 0]$ where $r<0$ and $s<0$, then the transformation $x=-x^{\prime}, y=-y^{\prime}, z=z^{\prime}$ of determinant one transforms $f$ into $[a, b, c,-r,-s, 0]$. Similar statements may be made if $r<0$ and $s>0$ or $r>0$ and $s<0$. Similar remarks apply when $f=[a, b, c, r, 0, t]$. 
Corollary. Let $f_{1}$ be given as in Theorem 1 . If $g=1$, then $f_{1}$ is equivalent to a reduced form $[a, b, c, r, s, t]$ where either $t=1$ and $s=0$ or $t=0$ and $s=1$.

Theorem 6. Any ternary quadratic form $f_{2}$ is equivalent to a unique reduced form.

Proof. By Theorem 1, $f_{2}$ is equivalent to a form $f_{1}=\left[a, b_{1}, c_{1}, r_{1}, s_{1}\right.$, $\left.t_{1}\right]$ in which $|a|=m_{1}$. Then Theorem 5 gives the desired conclusion since by Theorem $1, g_{1} \mid g$.

EXAMPLE 1. $f_{1}=3 x_{1}^{2}+3 y_{1}^{2}+3 z_{1}^{2}-2 y_{1} z_{1}-2 x_{1} z_{1}-2 x_{1} y_{1}$ with determinant $d=16$ is a reduced form according to Eisenstein's definition. What is the equivalent reduced form according to our definition?

Now $\phi=3 f_{1}=X^{2}+2 Y^{2}+6 z_{1}^{2}, \quad X=3 x_{1}-y_{1}-z_{1}, \quad Y=2 y_{1}-z_{1}$. Thus $\phi( \pm 1, \pm 1,0)=3$. But $x_{1}$ and $y_{1}$ are not integers. Hence $f_{1} \neq 1$. Likewise $f_{1} \neq 2$. Thus $f_{1}(1,0,0)=3$ is the first minimum of $f_{1}$. Also $f_{1}(0,1,0)$ $=3$ is the second minimum of $f_{1}$. Hence by (7)

$$
T=\left(\begin{array}{lll}
1 & 0 & \sigma \\
0 & 1 & \beta \\
0 & 0 & \delta
\end{array}\right),|T|=1
$$

where $\rho=0, \alpha=1, \gamma=0$ with $\sigma, \beta$ and $\delta$ yet to be determined. Thus $1=|T|=\delta$. By (2) $g_{1}=(3,-1,-1)=1$. Hence by (6) of Theorem 2, $t= \pm 1$ and $s=0$ since by $(8)_{4} t=-1$ holds. According to our definition we choose $s=0$ in $(8)_{5}$. Thus we seek the minimum value of $f_{1}(\sigma, \beta, 1)$ where $\sigma$ and $\beta$ are integral solutions of $3 \sigma-\beta-1=0$. Now all solutions of this equation are given by $\sigma=1-n$ and $\beta=2-3 n$, where $n$ is an arbitrary integer. Hence $f_{1}(1-n, 2-3 n, 1)=6(2 n-1)^{2}+2 \Rightarrow f_{1}(1,2,1)$ $=8$ is a third minimum. Thus applying the transformation $X_{1}=x+z$, $y_{1}=y+2 z$ and $z_{1}=z$ we obtain the equivalent form $g=3 x^{2}+3 y^{2}+8 z^{2}$ $+8 y z-2 x y$. But replacing $y$ by $-y$ and $z$ by $-z$ in $g$ replaces $g$ by the reduced form $f=3 x^{2}+3 y^{2}+8 z^{2}+8 y z+2 x y$. Note that $f_{1}(0,-1,1)$ $=8$. Thus the transformation $x_{1}=x, y_{1}=y-z$ and $z=z$ applied to $f_{1}$ gives the equivalent form $g=3 x^{2}+3 y^{2}+8 z^{2}-8 y z-2 x y$. Replacing $x$ by $-x$ and $z$ by $-z$ gives $f$.

EXAMPLE 2. Let $f_{2}=3 x_{2}^{2}+7 y_{2}^{2}+43 z_{2}^{2}+2 y_{2} z_{2}+12 x_{2} z_{2}+8 x_{2} y_{2}$ with determinant $d=8$. Find the reduced form equivalent to $f_{2}$.

By completing the squares in $x_{2}, y_{2}$ and $z_{2}$, we find the first minimum of $f_{2}$ to be given by $f_{2}(-7,4,1)=2$. Thus in (3) take $k_{1}=-7, l_{1}=4$ and $n_{1}=1$. Hence $g_{3}=(-7,4)=1$. Thus $l_{2}=1, k_{2}=-2$ is a solution of $-7 l_{2}-4 k_{2}=1$. In $n_{3}-k_{3}=1$, take $n_{3}=1$ and $k_{3}=0$. Hence 


$$
T^{\prime}=\left(\begin{array}{rrr}
-7 & -2 & 0 \\
4 & 1 & 0 \\
1 & 0 & 1
\end{array}\right), \quad\left|T^{\prime}\right|=1,
$$

takes $f_{2}$ into $f_{1}=[2,3,43,-11,5,-1]$. Thus $\phi=10 f_{1}=5 X^{2}+Y^{2}$ $+16 z_{1}^{2}$ where $X=2 x-y_{1}+5 z_{1}$ and $Y=5 y_{1}-17 z_{1}$. Hence we find that $X=0, Y=2$ and $z_{1}=-1 \Rightarrow x=1, y_{1}=-3$, and $z_{1}=-1$ so that $f_{1}(1,-3,-1)=2$ is a possible second minimum. By $(2), g=(a, d)$ $=(2,8)=2$ and $g_{1}=\left(a, t_{1}, s_{1}\right)=(2,-1,5)=1$. From $(8)_{2}$ and $f_{1}(1,-3,-1)$ we conclude that $\rho=1, \alpha=-3, \gamma=-1$. From (4) and (5) $a^{\prime} \rho+t^{\prime} \alpha+s^{\prime} \gamma=2(1)+(-1)(-3)+5(-1)=0$ so that $f_{1}(1,-3,-1)=2$ is a second minimum provided (27) holds; i.e., if $a[\alpha \sigma+(\alpha \rho) n-\rho \beta]=2[-3 \sigma-3 n-\beta]=\alpha s-s_{1}=-3 s-5$, where $s=1$. Thus (27) has a solution in $\sigma$ and $n$ if we take $\beta=1$ such that $1=|T|=\alpha \delta-\beta \gamma=-3 \delta+1$. Thus $\delta=0$. Now (27) reduces to

$$
\sigma+n=1 \text {. }
$$

All solutions of (39) are given by

$$
\sigma=k, \quad n=1-k
$$

where $k$ is an arbitrary integer. All solutions of $|T|=1$, i.e. of $-3 \delta$ $+\beta=1$ are given by

$$
\delta=n, \quad \beta=1+3 n
$$

where $n$ is an arbitrary integer. By (40) and (41)

$$
\sigma=k, \quad \beta=4-3 k, \quad \delta=1-k,
$$

where $k$ is to be chosen so that $f_{1}(k, 4-3 k, 1-k)$ is a third minimum. Now $f_{1}(k, 4-3 k, 1-k)=2 k^{2}-2 k+3 \Rightarrow 2 f_{1}=(2 k-1)^{2}+5$. Take $k=1$ and hence by $(42), f(1,1,0)=3$. By (7)

$$
T=\left(\begin{array}{rrr}
1 & 1 & 1 \\
0 & -3 & 1 \\
0 & -1 & 0
\end{array}\right)
$$

takes $f_{1}$ into the reduced form $f=2 x^{2}+2 y^{2}+3 z^{2}+2 y z+2 x z$. If we had chosen $k=0$, then $T$ with its 3 rd column replaced by $(0,4,1)$ takes $f_{1}$ into $[2,2,3,-1,1,0]$ which is equivalent to $f$ by replacing $x$ by $-x$ and $z$ by $-z$.

EXAMPLE 3. Let $f_{2}=2 y z+2 x z+2 x y$ with determinant $d=2$. Find the reduced form $f$ equivalent to $f_{2}$. 


$$
m_{1}=a=f_{2}(1,1,0)=2 .
$$

By Theorem 1, we may take

$$
T^{\prime}=\left(\begin{array}{lll}
1 & 1 & 0 \\
1 & 2 & 0 \\
0 & 0 & 1
\end{array}\right) .
$$

Thus $T^{\prime}$ transforms $f_{2}$ into $f_{1}=[2,4,0,3,2,3]$. By $(2) g=(2,2)=2$ and $g_{1}=(2,3,2)=1$. By (8) and Theorems 4 and 5 , we choose $\rho, \alpha$ and $\gamma$ such that $\left|f_{1}(\rho, \alpha, \gamma)\right|=|b|=m_{2}$, a second minimum, and where by (8), $2 \rho+3 \alpha+2 \gamma=0$. Now $2 f_{1}=X^{2}-Y^{2}-4 z^{2}$ where $X=2 x+3 y$ $+2 z$, and $y=Y$. Thus we take $x=\rho, y=\alpha$, and $z=\gamma$ with $X=Y=0$ and $z=-1$ so that $f_{1}=-2=b$. Then $2 \rho+3 \alpha=2$ holds with $\alpha=0$ and $\rho=1$. Note that $\gamma=z=-1$. Thus (36) holds for $2(1)=0 / 1-2(-1)$, $\beta \gamma=-1 \Rightarrow \beta=1$ and $3(1) \equiv 1 / 1\left(\bmod d_{3}\right)$ where, by $(30), d_{3}=(2,2)=2$ and $s$ is taken $=g_{1}=1$. It remains to choose $\sigma$ and $\delta$ in (7) so that $|c|=\left|f_{1}(\sigma, 1, \delta)\right|=m_{3}$ and such that $(8)_{5}$ holds, i.e., $2 \sigma+3(1)+2 \delta=s$ where $s=1$. Thus we choose $\sigma$ and $\delta$ so that $\sigma=-(\delta+1)$ and $m_{z}$ $=\left|f_{1}[-(\delta+1), 1, \delta]\right|=\left|-2 \delta^{2}\right| \geqq m_{2}=2$. Hence take $\delta=-1$ so that $\sigma=0$. Thus by (7)

$$
T=\left(\begin{array}{rrr}
1 & 1 & 0 \\
0 & 0 & 1 \\
0 & -1 & -1
\end{array}\right)
$$

transforms $f_{1}$ into $f=[2,-2,-2,-2,1,0]$ with determinant $d=2$ which is equivalent to the reduced form $[2,-2,-2,2,1,0]$.

\section{REFERENCES}

1. L. E. Dickson, Modern elementary theory of numbers, Univ. of Chicago Press, Chicago, Ill., 1939, pp. 90-92, Theorem 81. MR 1, 65.

2. - Studies in the theory of numbers, Univ. of Chicago Press, Chicago, Ill., 1930, p. 24, Theorem 26.

3. E. H. Hadlock and Amin Muwafi, On the equivalence of a ternary quadratic form, Proc. Amer. Math. Soc. 19 (1968), 1167-1168. MR 38 \#117.

University of Florida, Gainesville, Florida 32601 